\title{
Woodland strawberry WRKY71 acts as a promoter of flowering via a transcriptional regulatory cascade
}

\author{
Yingying Lei ${ }^{1}$, Yiping Sun ${ }^{1}$, Baotian Wang ${ }^{1}$, Shuang Yu ${ }^{1}$, Hongyan Dai ${ }^{1}, \mathrm{He} \mathrm{Li}^{1}$, Zhihong Zhang ${ }^{1}$ and Junxiang Zhang ${ }^{1}$
}

\begin{abstract}
The WRKY proteins are a large family of transcription factors that play important roles in stress responses and plant development. However, the roles of most WRKYs in strawberry are not well known. In this study, FvWRKY71 was isolated from the woodland strawberry 'Ruegen'. FVWRKY71 was highly expressed in the shoot apex and red fruit. Subcellular localization analysis showed that FVWRKY71 was located in the nucleus. Transactivation analysis showed that FvWRKY71 presented transcriptional activation activity in yeast. Overexpression of FvWRKY71 in Arabidopsis and woodland strawberry revealed early flowering in the transgenic plants compared with the wild-type control. Gene expression analysis indicated that the transcript levels of the flowering time and development integrator genes AP1, LFY, FT, AGL42, FUL, FPF1, SEP1, SEP2, and SEP3 were increased in FVWRKY71-overexpressing Arabidopsis and strawberry plants compared with the wild-type controls, which may result in accelerated flowering in transgenic plants. Furthermore, FVWRKY71 was proven to directly bind to the W-boxes (TTGACT/C) of the FVFUL, FVSEP1, FVAGL42, FVLFY, and FVFPF1 promoters in vitro and in vivo. Taken together, our results reveal a transcriptional regulatory cascade of FVWRKY71 involved in promoting flowering in woodland strawberry.
\end{abstract}

\section{Introduction}

The WRKY proteins are a family of plant transcription factors (TFs). Members of the family harbor one or two conserved WRKY domains, which contain a conserved WRKYGQK heptapeptide sequence at the N-terminus and a $\mathrm{C}_{2} \mathrm{H}_{2}$ or $\mathrm{C}_{2} \mathrm{HC}$ zinc-finger protein motif at the Cterminus ${ }^{1}$. Based on the number of WRKY domains and the structure of the C-terminal zinc-finger motif, the WRKY family is divided into three categories: groups I, II, and III. Group II proteins are further classified as IIa, IIb, IIc, IId, or IIe proteins based on the primary amino acid sequence $^{2}$. It has been more than 20 years since the identification of the first WRKY protein ${ }^{1}$. Since that time,

\footnotetext{
Correspondence: Junxiang Zhang (jxzhang@syau.edu.cn)

'Liaoning Key Laboratory of Strawberry Breeding and Cultivation, College of Horticulture, Shenyang Agricultural University, 120 Dongling Road, Shenyang 110866, China

These authors contributed equally: Yingying Lei, Yiping Sun
}

diverse biological functions of WRKY proteins in different species have been studied ${ }^{3}$.

WRKY proteins play crucial roles in plant signaling, the regulation of plant development and the responses to various biological and abiotic stresses ${ }^{4}$. In Arabidopsis, most WRKY genes have been shown to be involved in defense against pathogens ${ }^{2}$. For example, AtWRKY33 can modulate host defenses against Alternaria brassicicola and Botrytis cinerea infection ${ }^{5,6}$. AtWRKY46 forms a complex with AtWRKY70 and AtWRKY53 to positively regulate basal resistance to Pseudomonas syringae ${ }^{7}$. AtWRKY8, AtWRKY25, AtWRKY33, and AtWRKY63 are involved in the response to abiotic stress ${ }^{8-11}$. AtWRKY6, AtWRKY44, AtWRKY57, and AtWRKY71 are involved in plant developmental processes ${ }^{2,12-14}$. In rice, at least five OsWRKY genes have been demonstrated to participate in the defense response against pathogens ${ }^{15,16}$. In addition, many OsWRKY genes are regulators of the response to abiotic stresses, such as heat, drought, and salt ${ }^{16-18}$. Most

\section{(c) The Author(s) 2020}

(c) (i) Open Access This article is licensed under a Creative Commons Attribution 4.0 International License, which permits use, sharing, adaptation, distribution and reproduction cc) in any medium or format, as long as you give appropriate credit to the original author(s) and the source, provide a link to the Creative Commons license, and indicate if changes were made. The images or other third party material in this article are included in the article's Creative Commons license, unless indicated otherwise in a credit line to the material. If material is not included in the article's Creative Commons license and your intended use is not permitted by statutory regulation or exceeds the permitted use, you will need to obtain permission directly from the copyright holder. To view a copy of this license, visit http://creativecommons.org/licenses/by/4.0/. 
WRKY genes are involved in the stress response, but some participate in the determination of flowering time. In Arabidopsis, WRKY75 positively regulates flowering via the GA-mediated signaling pathway ${ }^{19}$. AtWRKY12 is a positive factor in the regulation of flowering, whereas AtWRKY13 is a negative factor in the modulation of flowering under short-day conditions. Further analysis indicated that both WRKY12 and WRKY13 can directly bind to the promoter of $F U L$ and produce different effects on their downstream target genes ${ }^{20}$. The heterologous overexpression of WRKY12 in Miscanthus lutarioriparius in Arabidopsis promotes flowering. Consistent with the early flowering phenotype, the expression levels of the APETALA 1 (AP1), FLOWERING LOCUS T (FT), LEAFY $(L F Y$ ), and FRUITFULL (FUL) genes are significantly increased in transgenic Arabidopsis plants ${ }^{21}$. AtWRKY71 accelerates flowering by regulating the flowering genes $F T$ and $L F Y^{22}$. The overexpression of CpWRKY71 in Arabidopsis also causes an early flowering, precocious leaf senescence phenotype ${ }^{23}$. The potential pathways of the WRKY TFs involved in flowering are not fully understood.

The molecular mechanism of flowering has been extensively studied in the annual model plant Arabidopsis, whereas less is known about the molecular control of flowering in perennial species, such as strawberry. Strawberry plants can be divided into two main groups based on their flowering habits: seasonal flowering (SF) and perpetual flowering (PF) strawberry. SF strawberry flowers under short days and is induced to flower in autumn ${ }^{24}$. SF patterns exist in diploid woodland strawberry (Fragaria vesca) and cultivated strawberry (Fragaria $\times$ ananassa), which also exhibit genomes with a high degree of colinearity ${ }^{25-27}$. In $F$. vesca, SF is caused by a single repressor gene: SEASONAL FLOWERING LOCUS $(S F L)^{28}$. TERMINAL FLOWER1 (TFL1), a candidate gene for $S F L$, is activated to inhibit strawberry flowering in summer and is suppressed to induce flower initiation in autumn $^{29}$. The knockdown of FvSOC1 produces a continuous flowering phenotype, which is similar to mutant of the floral repressor $F v T F L 1^{30}$. In SF strawberry, FvFT1 and FaFT1 are homologous genes of FT in Arabidopsis. PF strawberry produces new inflorescences continuously throughout the growing season from spring until late autumn. In the diploid Fragaria vesca, the PF trait is caused by a recessive mutation in the $S F L$ gene $^{31}$. A major locus controlling PF in cultivated strawberry is the perpetual flowering and runnering (PFRU) locus, which modulates the balance between sexual and asexual plant reproduction $^{27,31}$. FaPFRU shows opposite effects on flowering and runnering, exerting a positive effect on flowering and a negative effect on runnering, indicating that the two traits are genetically linked and share common physiological control ${ }^{31}$.
To date, the only available information on the WRKY family in woodland strawberry has come from the genome-wide analysis of the expression of WRKY genes in different developmental stages or under biotic and abiotic stresses ${ }^{32,33}$ and the potential roles of the WRKY genes in woodland strawberry are largely unknown. In the annual model plant Arabidopsis, WRKY71 can promote flowering. However, whether WRKY71 presents a similar function or regulatory mechanism in flowering in perennial plants, such as strawberries, is still largely unknown. In addition, the expression of FvWRKY46, another name of $F v W R K Y 71$, is upregulated during fruit development and ripening based on RNA-seq and RTqPCR results ${ }^{32}$, which indicates that $F v W R K Y 71$ may play a role in regulating fruit development and ripening, in addition to promoting flowering. To investigate the role of WRKY71 in $F$. vesca, the FvWRKY71 gene was overexpressed in Arabidopsis and woodland strawberry, and we found that FvWRKY71 conferred early flowering. We also found that FvWRKY71 accelerated flowering through the direct activation of different flowering-related genes between strawberry and Arabidopsis. These data will enrich the regulatory network of WRKY71 in different plants and provide some information on the promotion of flowering in Rosaceae plants.

\section{Results \\ Structural analysis of FvWRKY71}

To investigate the function of the FvWRKY71 protein, we first identified and cloned FvWRKY71 from the woodland strawberry 'Ruegen' by RT-PCR using the specific primers shown in Supplemental Table S1. The coding sequence of FvWRKY71 was $1107 \mathrm{bp}$ in length, which encoded a protein of 368 amino acids. The estimated molecular weight and isoelectric point were 41.62 and $6.46 \mathrm{kDa}$, respectively.

To examine the evolutionary relationship between FvWRKY71 and its orthologs from multiple species, we performed multiple-sequence alignment using their fulllength amino acid sequences. A phylogenetic tree was constructed through neighbor-joining analysis (Fig. 1a). The phylogenetic tree results indicated that FvWRKY71 exhibited the closest genetic relationship to the WRKY71 proteins from apple and pear, which also belong to the Rosaceae family (Fig. 1a). FvWRKY71 was also homologous to Arabidopsis WRKY71, WRKY48, WRKY57, and WRKY23 according to BLAST analysis in the TAIR database.

To better understand the conserved domain of FvWRKY71, we used DNAMAN software to carry out amino acid sequence analysis. FvWRKY71 was shown to contain a conserved WRKYGQ domain and a conserved $\mathrm{C}_{2} \mathrm{H}_{2}$-type zinc-finger motif (Fig. 1b). Therefore, 
a

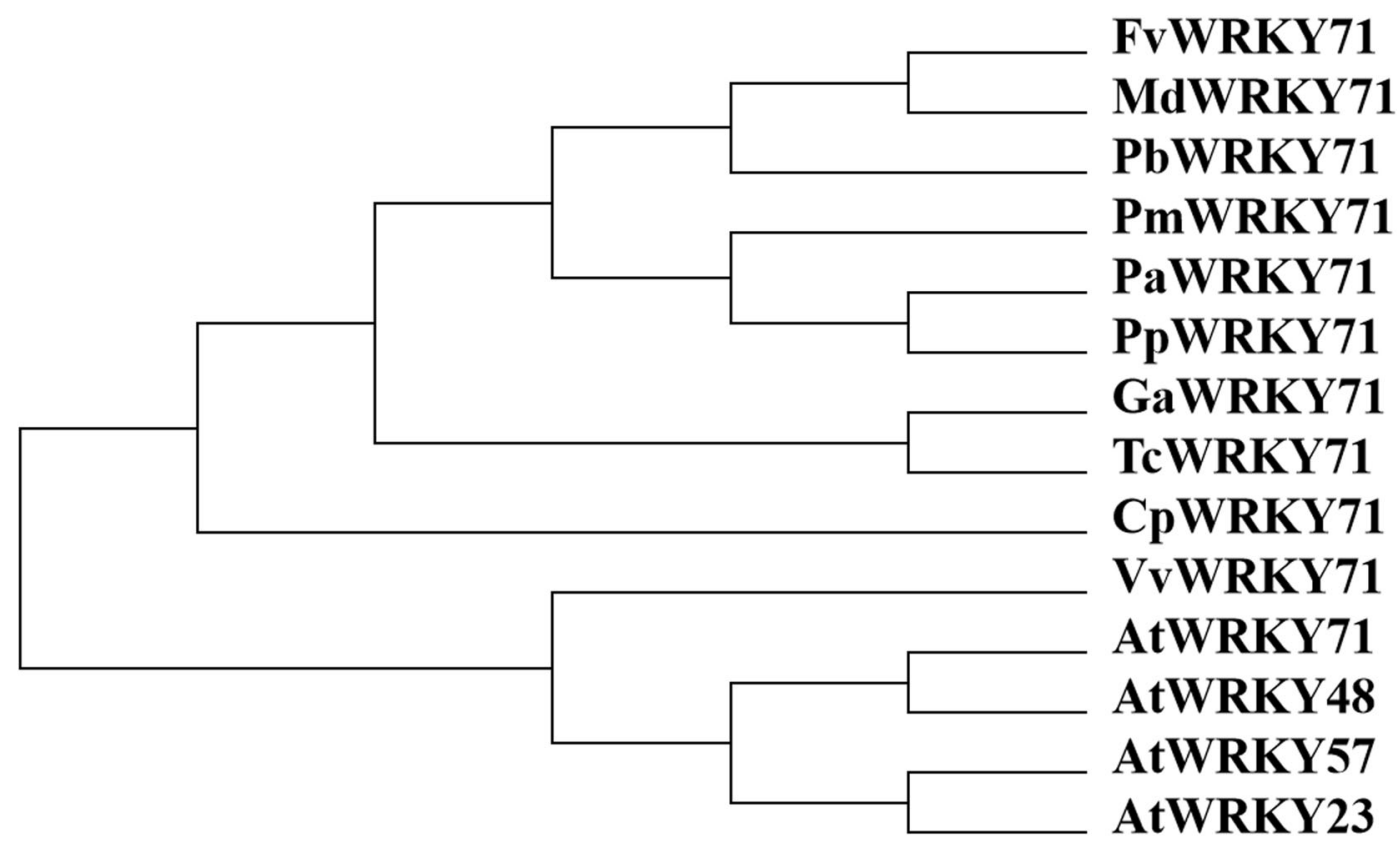

b

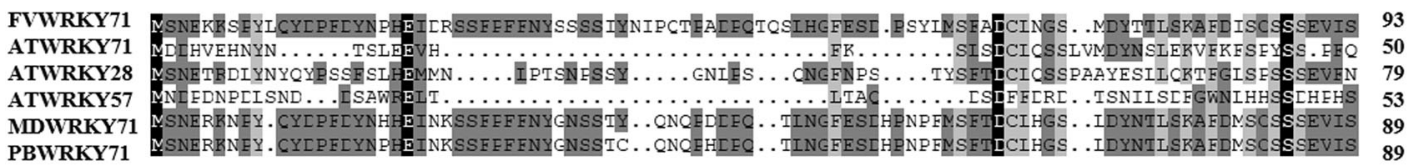

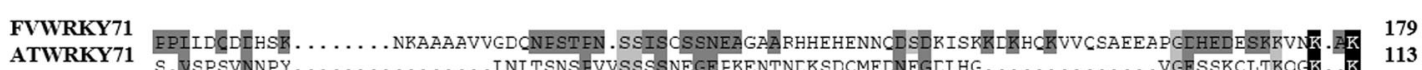

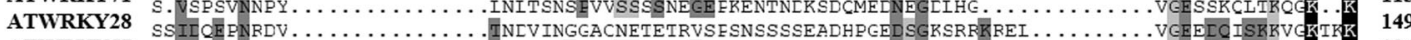
ATWRKY57

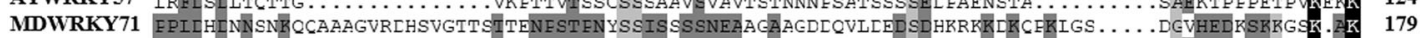

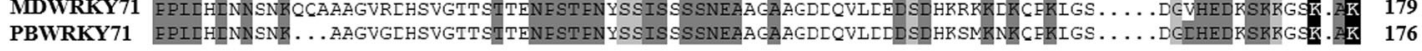

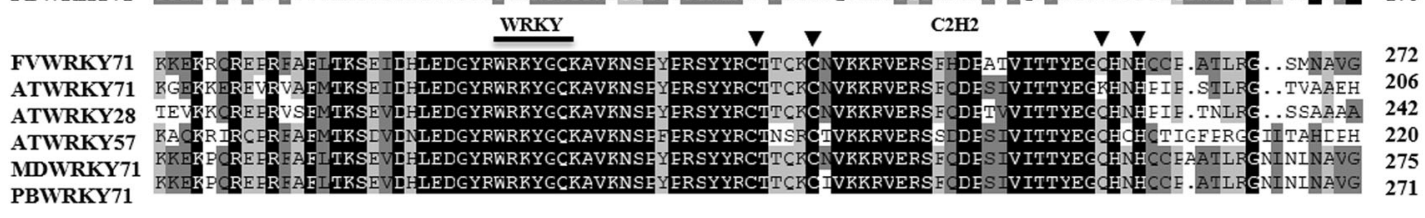

FVWRKY71

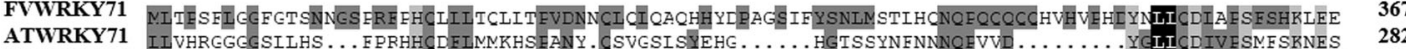

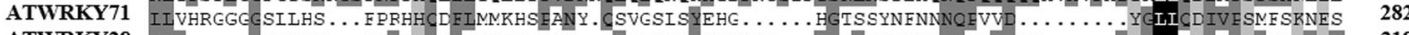

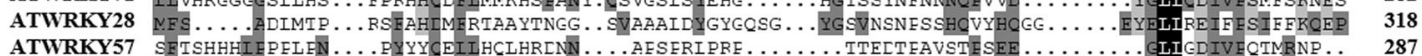

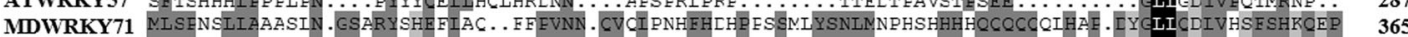

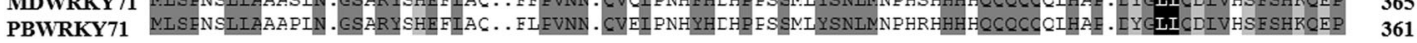

Fig. 1 Phylogenetic and structural analyses of FvWRKY71. a Phylogenetic tree based on the alignment of amino acid sequences among WRKY proteins. $\mathbf{b}$ Multiple alignment of WRKY proteins from higher plants

FvWRKY71 identified in this study contains one WRKY domain and belongs to group $\mathrm{II}^{34}$ (Fig. 1a).

\section{Expression patterns of FvWRKY71 in strawberry}

Different developmental stages and different organs were selected to detect the expression patterns of the FvWRKY71 gene by RT-qPCR (Fig. 2a). The expression levels in the flowers, shoot apex, and red fruits were approximately 2-, 6- and 17-fold higher than that in the roots, respectively. Lower expression levels were found in the roots, petioles, leaves, and green fruits. (Fig. 2a).

\section{Subcellular localization of FvWRKY71}

To examine the subcellular localization of the FvWRKY71 protein, we transiently expressed the recombinant FvWRKY71-GFP protein in Nicotiana benthamiana leaves. The fusion protein signal was examined by confocal microscopy. As shown in Fig. $2 b$, the 


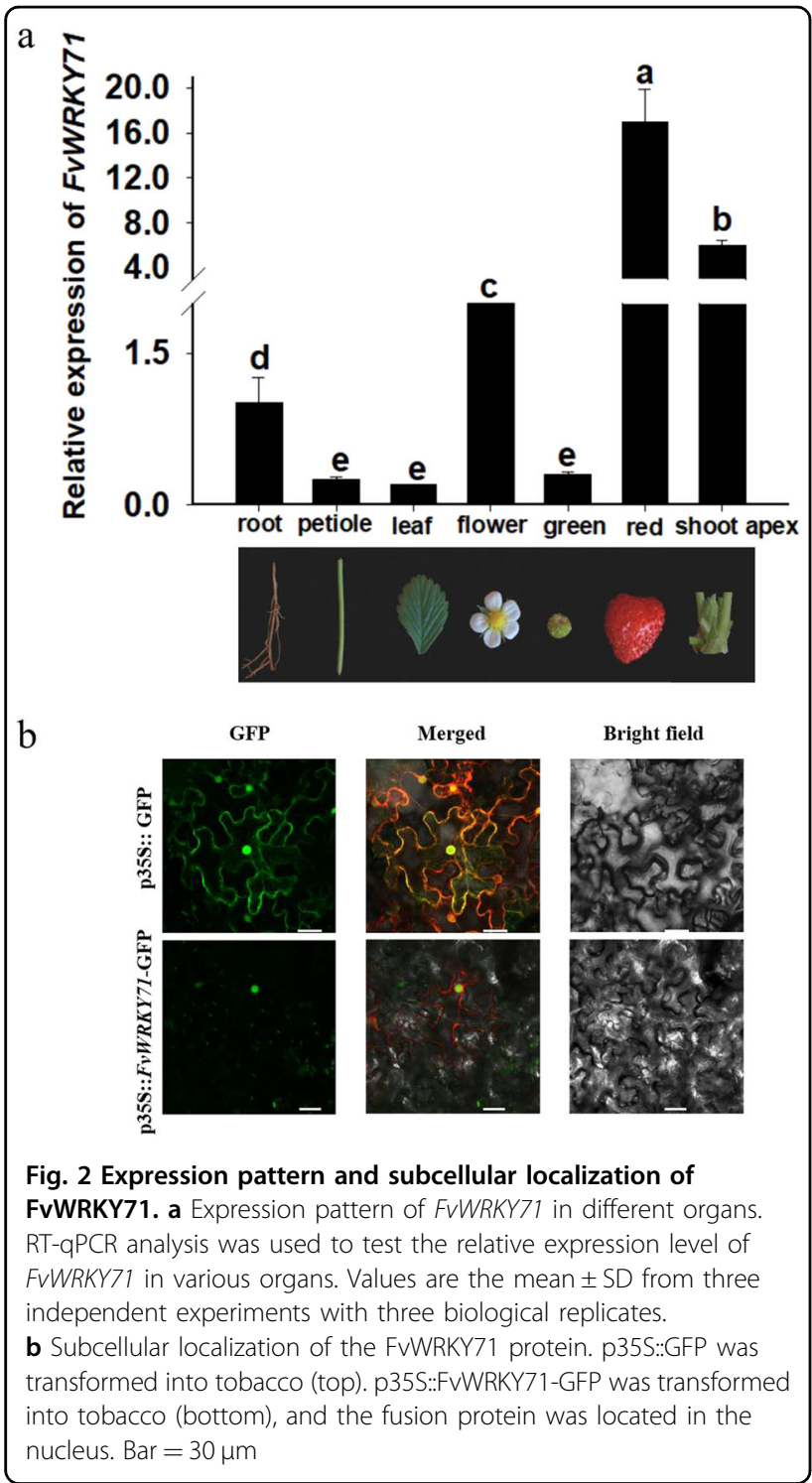

FvWRKY71-GFP fusion protein only existed in the nucleus, while the green signal of the GFP control was distributed in the nucleus and the cell membrane. The results indicate that FvWRKY71 localizes to the nucleus (Fig. 2b).

Transcriptional activation activity of FvWRKY71 in yeast

A yeast system was used to test whether FvWRKY71 exhibited transcriptional activation activity. The complete coding region of FvWRKY71 was fused to the GAL4 DNA-binding domain in the pGBT9 vector, and the construct was then transformed into Y2H Gold cells. The empty pGBT9 vector served as a control. As shown in Fig. 3a, yeast cells transformed with the pBD-FvWRKY71 fusion vector grew well on both $\mathrm{SD} /-\mathrm{Trp}$ and $\mathrm{SD} /-\mathrm{Trp} /-$ Leu/-Ade media. They also exhibited blue colonies on

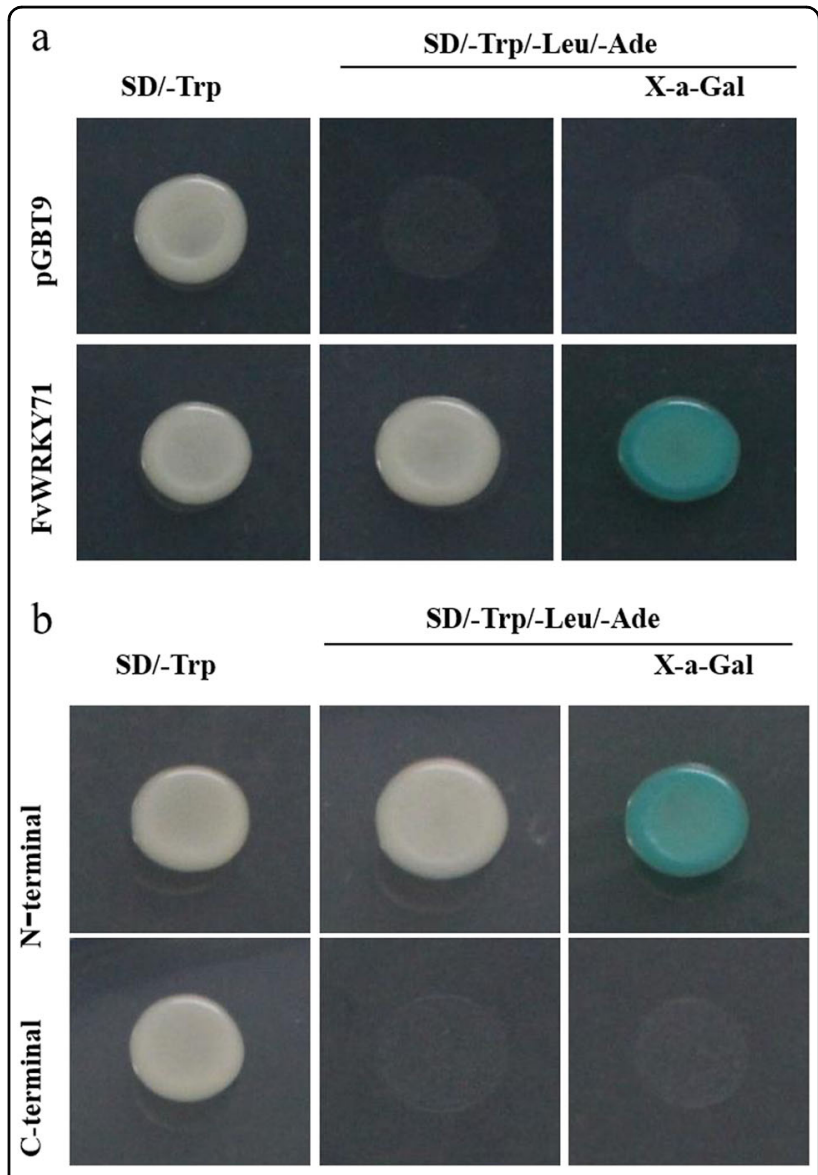

Fig. 3 Transactivation activation activation analysis of FvWRKY71. The construct of PBD-FvWRKY71 was transformed into yeast $\mathrm{Y} 2 \mathrm{H}$ Gold cells, and the cells were then examined on SD/-Trp and SD/-Trp/-Leu/-Ade/X-a-gal plates. a Transactivation activation analysis of FvWRKY71. b Transactivation activation analysis of the $\mathrm{N}$-terminus and C-terminus of FVWRKY71

alpha-galactosidase plates (Fig. 3a), which indicated that they presented alpha-galactosidase activity. In contrast, the control yeast strain was only viable on SD/-Trp medium. These data showed that FvWRKY71 exhibited transcriptional activation activity in yeast. To further elucidate which segments presented transcriptional activation activity, we transformed the $\mathrm{N}$-terminus and $\mathrm{C}$ terminus of FvWRKY71 in the pGBT9 vector into yeast cells. The results showed that the $\mathrm{N}$-terminus showed transcriptional activation activity, while the C-terminus did not (Fig. 3b).

\section{Overexpression of FvWRKY71 in Arabidopsis promotes flowering}

To investigate the biological function of the FvWRKY71 gene, we constructed a p35S::FvWRKY71 overexpression vector (Fig. S1a) and transformed it into Arabidopsis to obtain transgenic plants. The transgenic Arabidopsis lines 
were examined by PCR to determine on DNA levels (Fig. S1b) and by RT-qPCR to determine on RNA levels (Fig. 4c). Two $\mathrm{T}_{3}$ homozygous positive transgenic lines were selected for further phenotypic analysis. By observing transgenic lines 1 and 5, we found that the ectopic expression of the FvWRKY71 gene in Arabidopsis induced an early flowering phenotype compared with wild-type plants (Fig. 4a). Under long (16h) daylight conditions, FvWRKY71-overexpressing transgenic plants started bolting at 19 days after sowing, whereas wild-type plants started bolting at 33 days after sowing. In addition, the number of rosette leaves at flowering in the transgenic lines was approximately seven, while the number of rosette leaves at flowering in the wild-type plants was approximately thirteen (Fig. 4b). These data indicate that the overexpression of FvWRKY71 in Arabidopsis can promote flowering.

To investigate whether the overexpression of FvWRKY71 in Arabidopsis affected the transcript levels of flowering-related genes for the early flowering phenotype. We searched the Arabidopsis flowering database (http:// www.phytosystems.ulg.ac.be/florid/) for candidate genes responsible for accelerating flowering in FvWRKY71overexpressing transgenic lines. Twelve genes involved in the determination of flowering time and flower development were identified by RT-qPCR analysis. The expression levels of SEPALLATA1 (SEP1), SEPALLATA2 (SEP2), SEPALLATA3 (SEP3), AGMOUS-LIKE 42 (AGL42), AP1, LFY, FUL, FLOWERING PROMOTING FACTOR 1 (FPF1), and $F T$ were shown to be significantly increased compared with those in wild-type plants (Fig. 4c). The expression level of FvWRKY71 in transgenic line 5 was higher than that in transgenic line 1 , and the expression of downstream genes was also higher in transgenic line 5 than in transgenic line 1 (Fig. 4c).

\section{Overexpression of FvWRKY71 in woodland strawberry accelerates flowering}

To further investigate the function of FvWRKY71, we also overexpressed FvWRKY71 in 'Ruegen' strawberry. The transgenic lines were first examined by PCR to detect DNA levels (Fig. S1c). Next, we investigated transgenic lines 3 and 21 by RT-qPCR (Fig. 5c). The transcript level of $F v W R K Y 71$ was significantly increased in transgenic lines 3 and 21 compared with the non-transgenic control plants (Fig. 5c). Interestingly, we observed that the FvWRKY71-overexpressing transgenic lines promoted flowering in woodland strawberry relative to wild-type plants (Fig. 5a), and the early flowering phenotype was consistent with the results of $F v W R K Y 71$ overexpression in Arabidopsis. We found that transgenic line 3, transgenic line 21, and wild-type plants took 38,37 , and 55 days, respectively, to start flowering under the same conditions (Fig. 5b). Next, we tested the expression levels of SEP1, SEP2, SEP3, AGL42, AP1, LFY, FUL, SQUAMOSA PROMOTER BINDING PROTEIN-LIKE3 (SPL3), SPL4, FPF1, and FT in FVWRKY71-overexpressing strawberry transgenic lines by RT-qPCR. We found that the transcript abundance of these flowering-related genes was significantly increased in transgenic strawberry lines compared with wild-type control plants (Fig. 5c), which was in agreement with the results of the overexpression of FvWRKY71 in Arabidopsis (Fig. 5c). We also analyzed the expression of $F v S O C 1$ and FvTFL1 in transgenic strawberry plants by RT-qPCR. We found that the expression of $F v S O C 1$ and $F v T F L 1$ was significantly reduced in transgenic line 3 compared with that in wild-type plants, whereas there was no considerable difference between transgenic line 21 and wild-type plants (Fig. S3). Taken together, these data reveal that FvWRKY71 promotes flowering and regulates the expression of flowering-related genes.

\section{FvWRKY71 accelerates the flowering of woodland strawberry mainly via the direct activation of FvFUL, FVSEP1, FvAGL42, FvLFY, and FvFPF1}

To further identify the genes directly regulated by FvWRKY71 for early flowering in woodland strawberry, we investigated the promoter sequences of FvSEP1, FvSEP2, FvSEP3, FvAGL42, FvAP1, FvLFY, FvFUL, FvSPL3, FvSPL4, $F v F P F 1$, and $F v F T$ to determine whether they contained Wboxes (TTGACT/C). We found that FvFUL, FvSEP1, $F v A G L 42, F v L F Y$, FvFPF1, and FvSPL4 exhibit W-boxes in these promoter regions (Supplemental File S1). A yeast onehybrid experiment was first conducted to detect whether FvWRKY71 could bind to the promoters of FvFUL, FvSEP1, FvAGL42, FvLFY, FvFPF1, and FvSPL4. pAD-FvWRKY71 was cotransformed into yeast $\mathrm{Y} 1 \mathrm{H}$ strains in combination with proFvFUL-pAbAi, proFvSEP1-pAbAi, proFvAGL42pAbAi, proFvLFY-pAbAi, proFvFPF1-pAbAi, or proFvSPL4pAbAi. The first five transformed strains were able to grow on $\mathrm{SD}$ /-Leu selective medium containing aureobasidin A (ABA) $(100,100,150,300$, and $300 \mu \mathrm{g} / \mathrm{L}$, respectively), whereas that transformed with FvSPL4 $(100 \mu \mathrm{g} / \mathrm{L}$ ABA) could not (Fig. 6), which indicates that FvWRKY71 can bind to the promoters of FvFUL, FvSEP1, FvAGL42, FvLFY, and FvFPF1.

A luciferase assay is an important method for detecting the binding of transcription factors and specific domain sites in the promoters of target genes ${ }^{35}$. To clarify the effect of FvWRKY71 on the promoters of FvFUL, FvSEP1, $F v A G L 42, F v L F Y$, FvFPF1, and FvSPL4, we performed luciferase reporter assays in $N$. benthamiana leaf cells. We constructed proFvFUL-LUC, proFvSEP1-LUC, proFvAGL42-LUC, proFvLFY-LUC, proFvFPF1-LUC and proFvSPL4-LUC reporters and the effector plasmid 35S: FvWRKY71 (Fig. S1). As expected, FvWRKY71 exerted activation effects on FvFUL, FvSEP1, FvAGL42, FvLFY, and FvFPF1 in these LUC reporter assays, but not on 
a
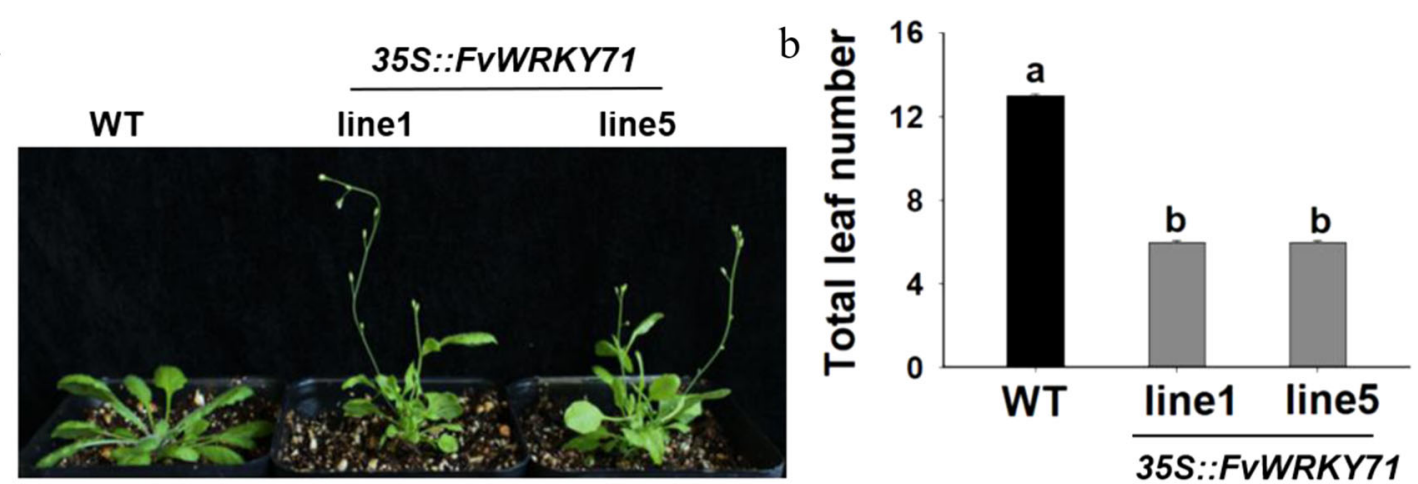

$\mathrm{C}$
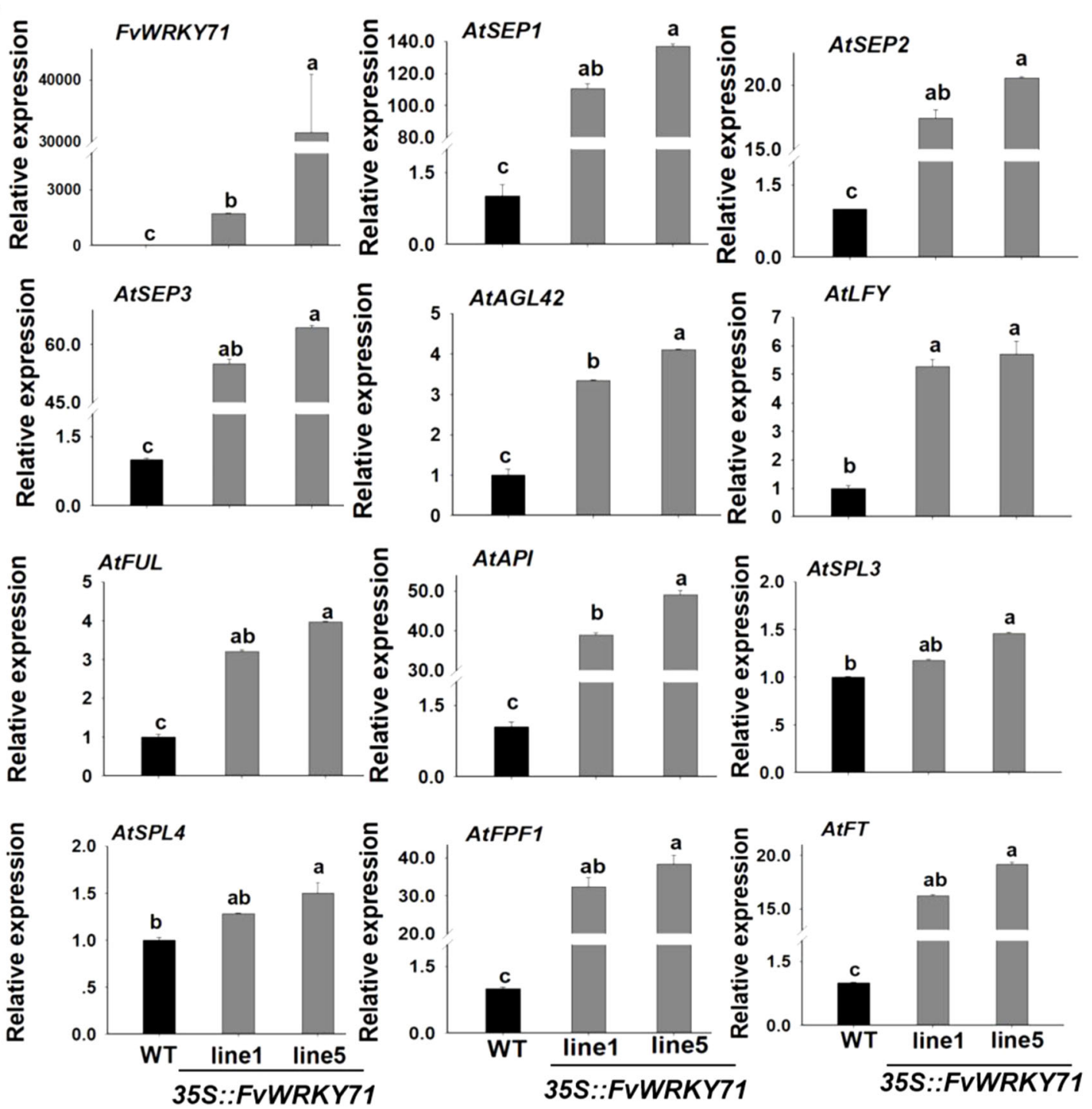

Fig. 4 The phenotypes of Arabidopsis FvWRKY71-overexpressing plants and gene expression analysis. a The phenotypes of Arabidopsis FvWRKY71-overexpressing transgenic lines and wild-type control plants. $\mathbf{b}$ The number of rosette leaves at flowering in transgenic plants and wildtype plants. c RT-qPCR analysis of FVWRKY71 and genes involved in flowering, including AtSEP1, AtSEP2, AtSEP3, AtAGL42, AtAP1, AtLFY, AtFUL, AtSPL3, AtSPL4, AtFPF1, and AtFT, between wild-type and transgenic Arabidopsis plants. Values are the mean \pm SD from three independent experiments with three biological replicates. Different letters indicate significant differences $(P<0.05$, based on Duncan's multiple range test) 
a

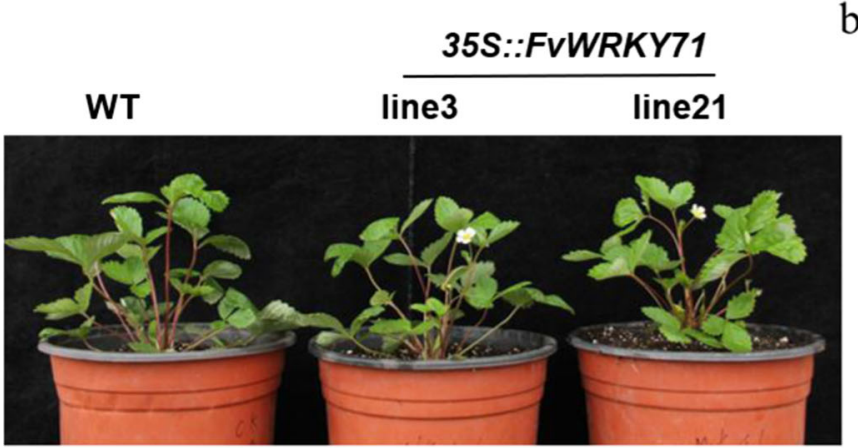

$\mathrm{C}$
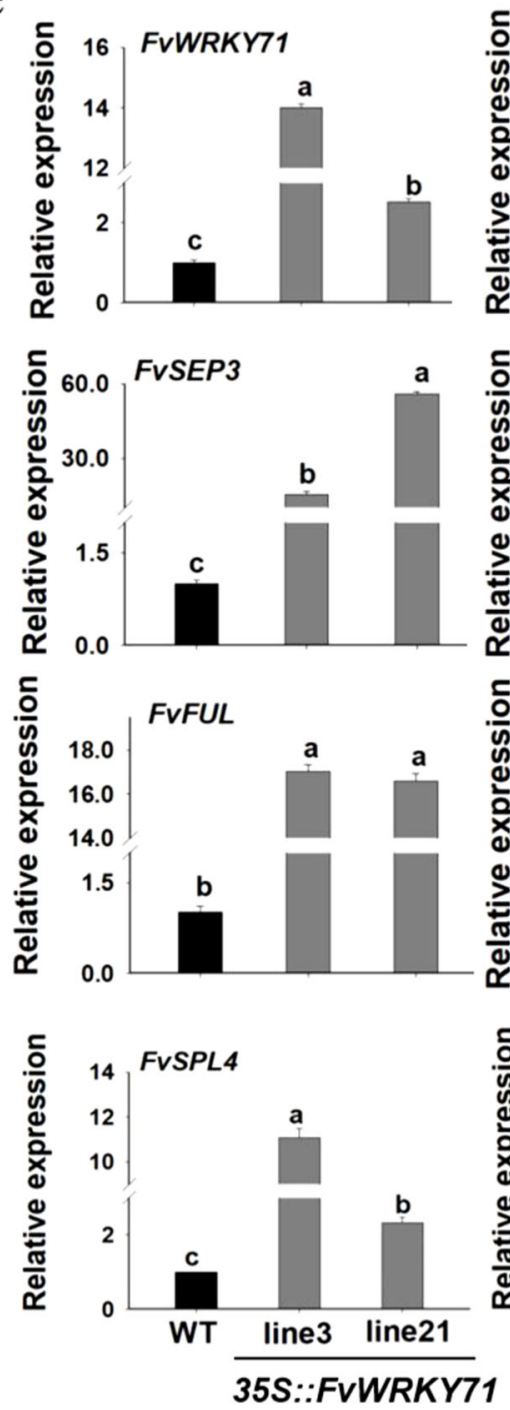

b

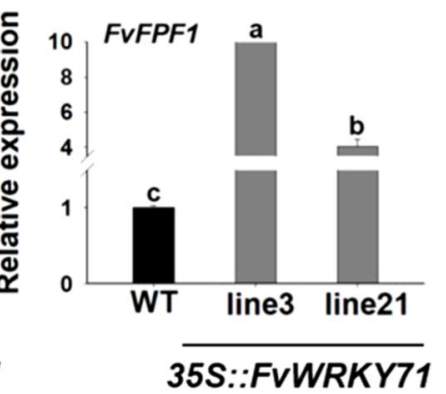

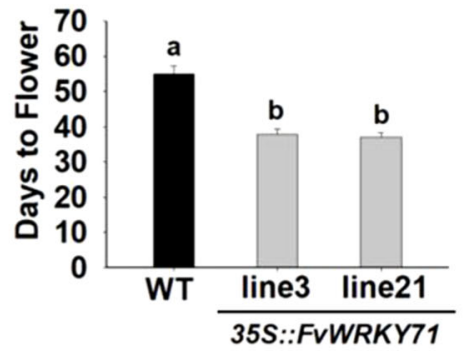
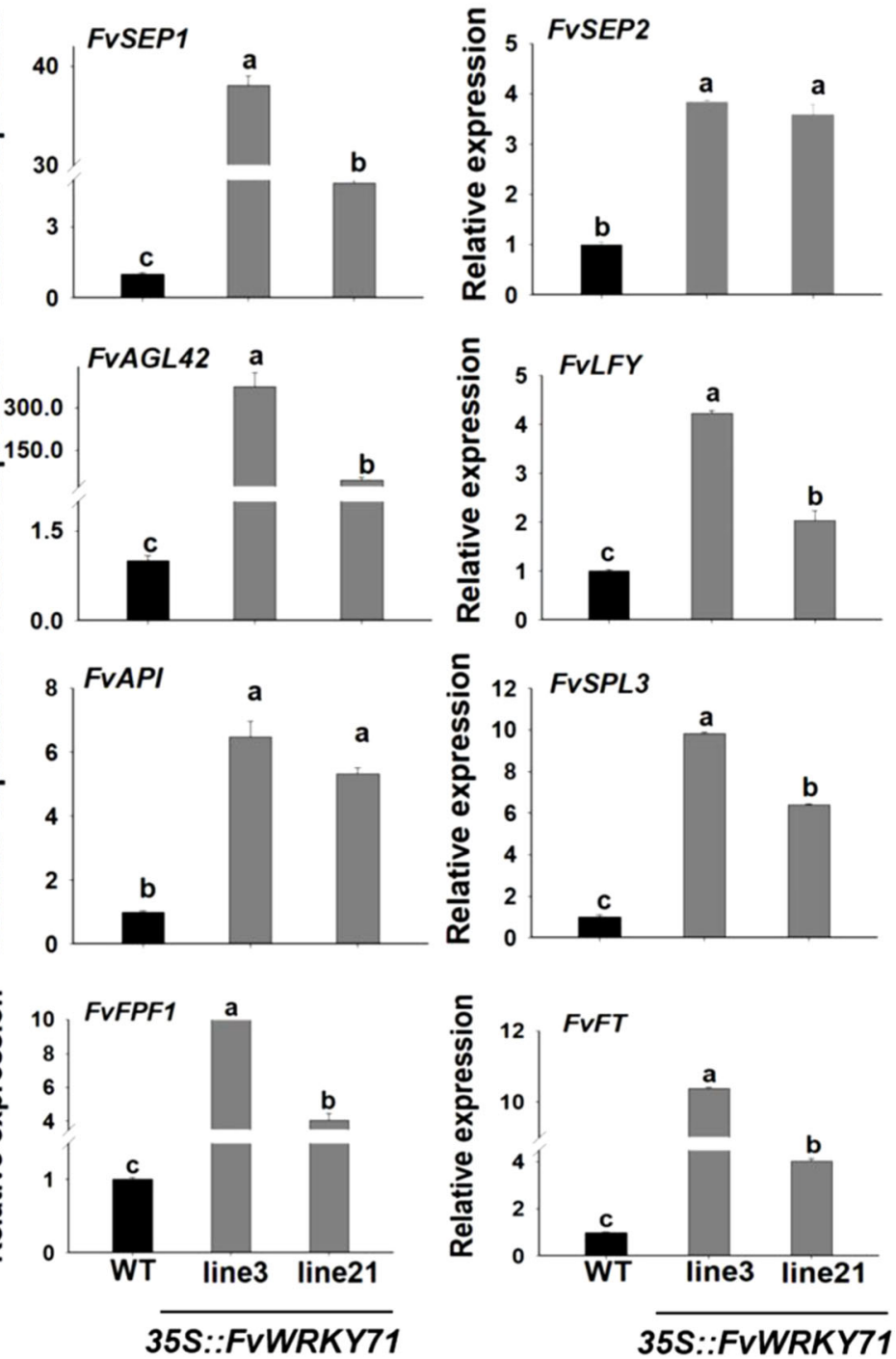

Fig. 5 The phenotypes of FvWRKY71-overexpressing transgenic woodland strawberry lines and gene expression analysis. a The phenotypes of FvWRKY71-overexpressing woodland strawberry lines and wild-type plants. $\mathbf{b}$ Days to flowering between woodland strawberry transgenic lines and wild-type plants after transfer from culture medium. c RT-qPCR analysis of FVWRKY71 and genes involved in flowering between wild-type and transgenic woodland strawberry plants, including FVSEP1, FVSEP2, FVSEP3, FVAGL42, FVAP1, FVLFY, FvFUL, FvSPL3, FvSPL4, FvFPF1, and FvFT. Values are the mean \pm SD from three independent experiments with three biological replicates. Different letters indicate significant differences $(P<0.05$, based on Duncan's multiple range test) 


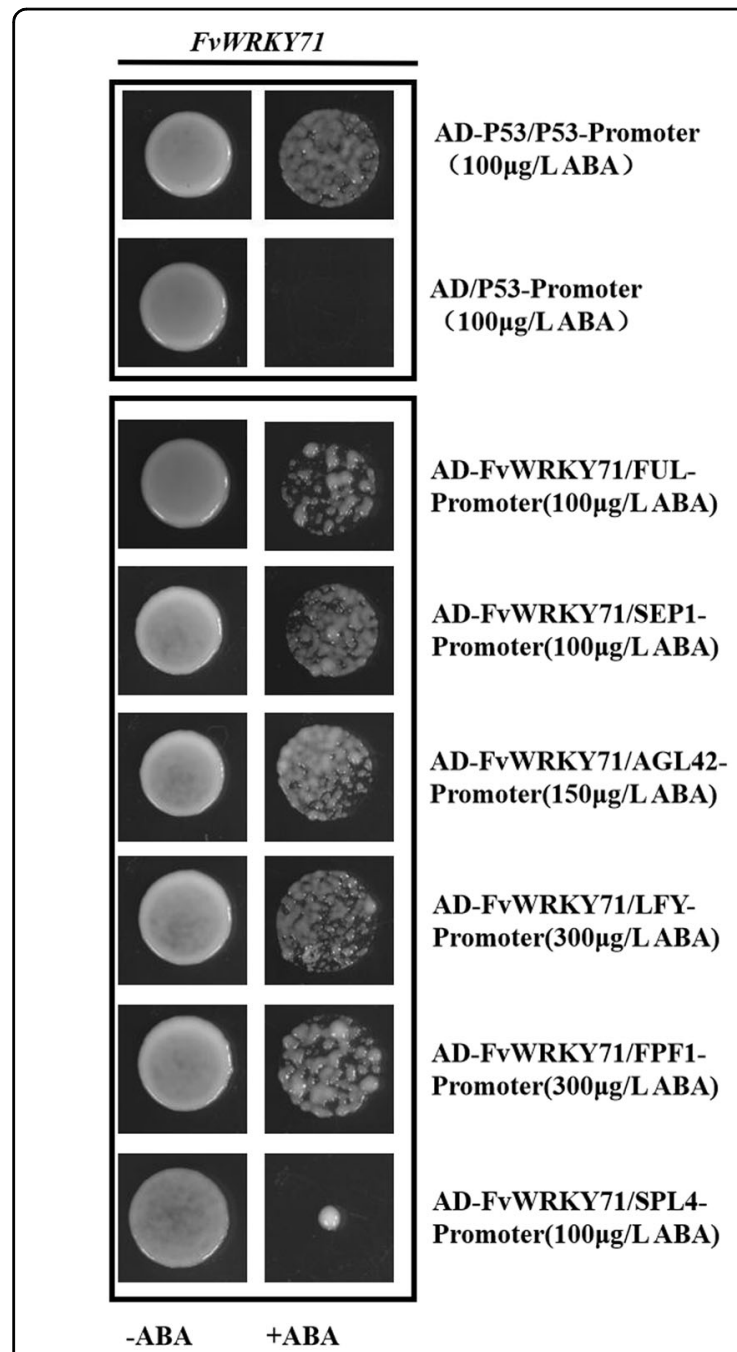

Fig. 6 Yeast one-hybrid assay to test whether FvWRKY71 could directly bind to the promoters of FvFUL, FVSEP1, FVAGL42, FVLFY, FvFPF1, and FvSPL4. pAD-FvWRKY71 was cotransformed into yeast $\mathrm{Y} 1 \mathrm{H}$ strains in combination with proFvFUL-pAbAi, proFvSEP1-pAbAi, proFvAGL42-pAbAi, proFvLFY-pAbAi, proFvFPF1-pAbAi, or proFvSPL4-pAbAi. The transformed strains all grew on SD/-Leu selective medium containing aureobasidin A (ABA) $(100,100,150,300,300$, and $100 \mu g / L$, respectively)

FvSPL4 (Fig. 7a), and FvWRKY71 activated the expression of the FvFUL, FvAGL42, FvSEP1, FvLFY, and FvFPF1 genes to various levels (7.4-, 4.0-, 5.4-, 9.9-, and 7.2-fold, respectively) compared with the corresponding control (Fig. 7b). Together, these data suggest that FvWRKY71 accelerates the flowering of woodland strawberry mainly through the direct activation of FvFUL, FvAGL42, FvSEP1, $F v L F Y$, and FvFPF1.

\section{Discussion}

Flowering is a key step in plant growth and development. Early flowering is an important trait for Rosaceae crops such as strawberry, apple, and peach. Here, we identified the transcription factor WRKY71 in woodland strawberry and found that FvWRKY71 was expressed in all examined organs, especially in the shoot apex and red fruit. In Arabidopsis, the transcript level of AtWRKY71 gradually increased with seedling development from 4 to 16 days and reached the highest level at 16 days, which is consistent with the floral transition ${ }^{22}$. The higher expression of FvWRKY71 in the shoot apex is consistent with the floral transition. Functional analysis by overexpressing FvWRKY71 in Arabidopsis and strawberry revealed that $F v W R K Y 71$ plays an important role in accelerating flowering.

Flower induction is crucial for plant growth and development, and can promote the shift from vegetative growth to reproductive growth ${ }^{36,37}$. Flower induction is regulated by many exogenous and endogenous factors. In the model plant Arabidopsis, at least five flowering pathways have been found, including the photoperiod, gibberellin, vernalization, autonomous, and age pathways ${ }^{38-40}$. These pathways are related to various signals, such as photoperiod, age, temperature, abiotic stress and hormones, and regulate the expression of factors involved in flowering. These flowering pathways can be regulated independently from each other or in an interrelated manner, thus forming a complex flowering regulation network. The functional analysis of genes representing various flowering pathways did not reveal large differences between diploid woodland strawberry and cultivated strawberry ${ }^{25,26,41}$.

In perennial plants, molecular studies on flowering have focused on the FT, LFY, TFL1, and SOC1 genes. As a key component of flowering pathways, the expression of $F T$ is influenced by many factors, some of which act as positive regulators and others as repressors ${ }^{42-47}$. The signal is passed on to FvAP1 and FvFUL to effect the transition from the vegetative to the reproductive stage $\mathrm{e}^{29,48}$. The $F T$ gene is highly expressed in the leaves in cultivated strawberry and is induced by long days and high temperatures $^{26}$. Decreasing or increasing $L F Y$ transcript abundance can delay or accelerate flowering, respectively $^{49}$. It has been reported that Arabidopsis WRKY75 can promote flowering via $F T$ and $S O C 1$ but not $L F Y$. The promoter of $F T$ contains some W-box cis-elements, and $F T$ is the direct target of AtWRKY75. In addition, AtWRKY75 partially controls GA signaling in the regulation of flowering time ${ }^{19}$. Arabidopsis WRKY12 and WRKY13 modulate flowering in opposite directions under short-day conditions. AtWRKY12 and AtWRKY13 can interact with two DELLA proteins, AtGAI and AtRGL1, to affect the transcriptional activity of AtWRKY12 and AtWRKY13, which regulate the expression of $A t F U L$, a direct downstream target gene of AtWRKY12 and AtWRKY13, to control flowering ${ }^{20}$. The 

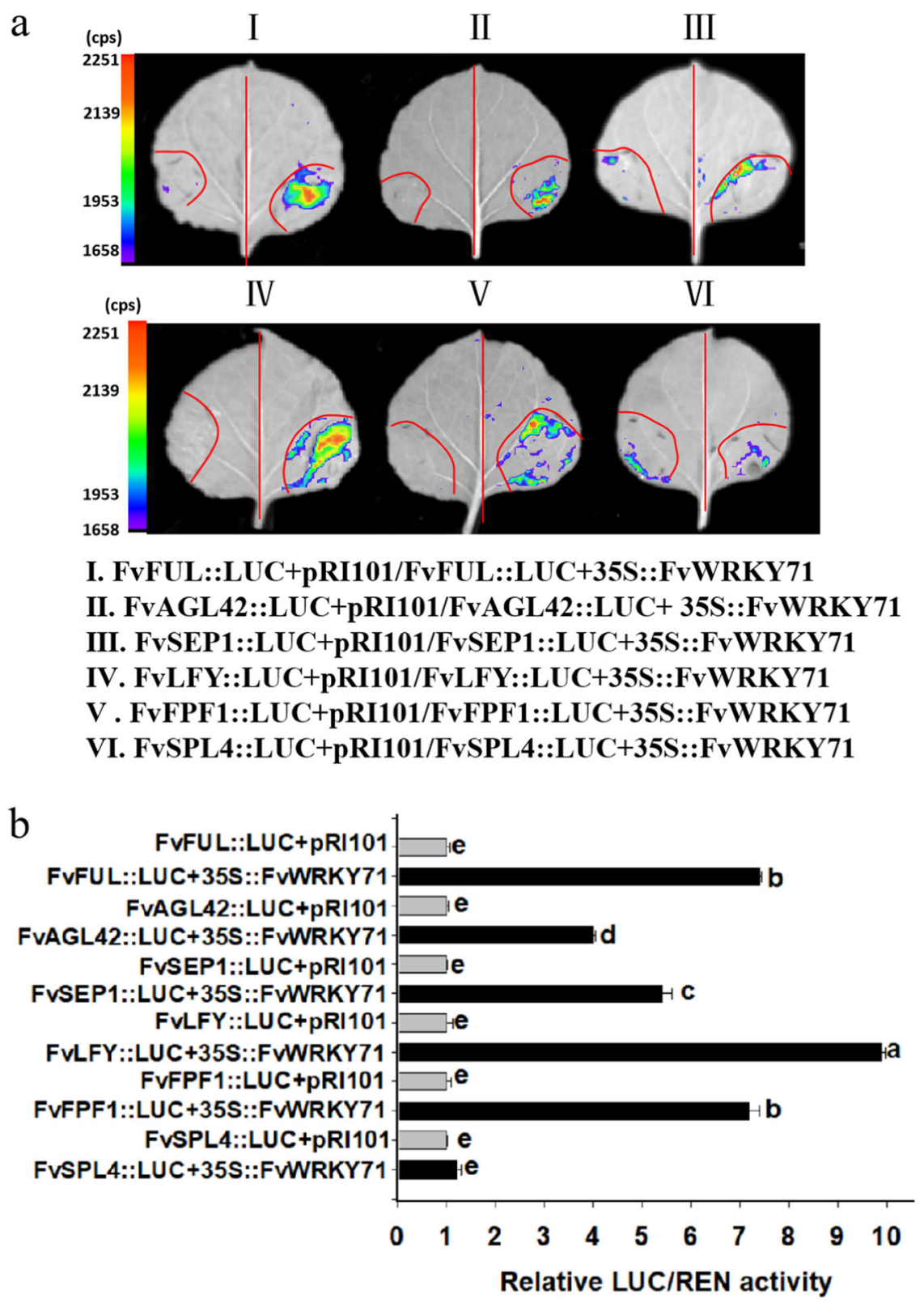

Fig. 7 Luciferase activity assay for determining whether FvWRKY71 could directly bind to the promoters of FvFUL, FvSEP1, FvAGL42, FvLFY, FvFPF1, and FvSPL4. a Tobacco transient expression assay. P35S.:FVWRKY71 was cotransformed into N. benthamiana in combination with proFvFUL:: LUC, proFvAGL42::LUC, proFvSEP1::LUC, proFVLFY::LUC, proFvFPF1::LUC, or proFvSPL4::LUC. b The comparison of luciferase activity. The transcriptional activity of these infiltrated tobacco leaves based on the ratio of LUC to REN was investigated with a dual luciferase reporter gene assay kit. Different letters indicate significant differences $(P<0.05$, based on Duncan's multiple range test)

transcription levels of $A P 1$ and $L F Y$ are higher in WRKY71-1D than in wild-type plants, and AtWRKY71 promotes flowering through the direct activation of $F T$ and $L F Y^{22}$. In our study, the relative expression levels of $F T, L F Y$, and FUL in the Arabidopsis and strawberry transgenic lines were also higher than those in the wildtype (Figs. 4c and 5c). We identified W-boxes in the $L F Y$ promoter region, while we could not find a W-box in the
FT promoter in woodland strawberry. There are three Wboxes in the Arabidopsis $F T$ promoter region ${ }^{22}$. Therefore, FvWRKY71 could not directly regulate $F T$ gene expression by binding to $\mathrm{W}$-box elements in woodland strawberry and might affect the transcript level of $F T$ in other unidentified ways. The overexpression of FUL in Arabidopsis can promote flowering ${ }^{50,51}$. Interestingly, we found that the strawberry FUL gene presented two 
W-boxes in its promoter region (Supplemental File S1) and showed that FvWRKY71 could interact with the FvFUL promoter to modulate FvFUL expression. These findings indicate that different WRKY proteins regulate the transcript levels of different floral meristem identity genes or floral integrators to control their distinct flowering pathways and that some WRKY proteins may be involved in controlling the timing of GA-mediated flowering.

TFL1 and SOC1 function as major repressors of flowering in perennial species, including strawberry and $\operatorname{rose}^{52-54}$. We found that the expression levels of FvTFL1 and FvSOC1 were significantly reduced in woodland strawberry transgenic line 3 compared with wild-type plants, which is consistent with the early flowering phenotype of FvWRKY71-overexpressing plants. However, there were no large differences between transgenic line 21 and wild-type plants (Fig. S3). The higher expression of FvWRKY71 in transgenic line 3 (14.0-fold higher than wild-type control) than transgenic line 21 (2.5-fold higher than wild-type control) may result in lower expression of FvTFL1 and FvSOC1 in transgenic line 3. In Arabidopsis, SUPPRESSOR OF OVEREXPRESSION OF CONSTANS1 (SOC1) mediates both endogenous and exogenous signals to accelerate flowering ${ }^{30}$. These findings reveal that the functions of important flowering genes may not be completely the same between annual and perennial plants.

During the transition to flowering, FPF1 regulates flowering through an independent pathway that is parallel to that of $L F Y$ and $A P 1^{55}$. We found $\mathrm{W}$-boxes in the promoter sequences of the FPF1 gene in woodland strawberry, and we showed that FvWRKY71 can directly regulate $F v F P F 1$ expression by binding to the $\mathrm{W}$-boxes of the FvFPF1 promoter. The SPL3 and SPL4 transcription factors can also directly bind to the promoter of the $L F Y$ gene $^{55}$. SPL3 directly activates $L F Y, F U L$, and $A P 1$ expression in Arabidopsis ${ }^{56}$. The expression levels of SPL3 and SPL4 are significantly increased in FvWRKY71overexpressing lines, and a W-box exists in the FvSPL4 promoter. However, FvWRKY71 cannot bind to the Wbox of the FvSPL4 gene (Figs. 6 and 7). In Arabidopsis, the promoter region of the AP1 gene contains five W-boxes, whereas AtWRKY71 cannot bind to any of the AP1 Wboxes $^{22}$. These findings indicate that the FVSPL4 and AtAP1 genes contain W-box elements but that they are not targets of WRKY71 in woodland strawberry and Arabidopsis. WRKY71 might regulate the expression of these genes to accelerate flowering in indirect ways.

MADS domain transcription factors play important roles in flower development, the control of flowering time and fruit development ${ }^{57}$. In our study, we observed that many MADS-domain proteins, such as AP1, AGL42, $S E P 1, S E P 2$, and $S E P 3$, exhibited different expression levels between FvWRKY71-overexpressing transgenic plants and wild-type plants (Figs. 4c and 5c). It has been reported that the MADS-domain proteins AP1, AP3, PI, AG, and SEP3 play important roles in floral tissues to modulate flower development ${ }^{58}$. SEP1, SEP2, and SEP3 encode MADS box transcription factors, which are a group of organ identity genes that are required for flower development. LFY directly activates $S E P 1, S E P 2$, and SEP3 in Arabidopsis ${ }^{59}$. AGL42 encodes MADS-box transcription factors and is closely related to SOC1 in Arabidopsis $^{60}$. Here, we found that the upstream regulatory region of woodland strawberry $A P 1$ does not contain a Wbox, while there are five $\mathrm{W}$-boxes in the Arabidopsis AP1 promoter. Additionally, we found that the promoter regions of FvAGL42 and FvSEP1 contain W-boxes (Supplemental File S1) and that FvWRKY71 is able to directly control the expression of FvAGL42 and FVSEP1 by binding to W-boxes in the promoters of FvAGL42 and FvSEP1 (Figs. 6 and 7). Therefore, these results indicate that FvWRKY71 promotes flowering through the direct activation of different flowering-related genes between strawberry and Arabidopsis.

Together, our results show that FvWRKY71 acts as a positive regulator of flowering in woodland strawberry. The FUL, SEP1, AGL42, LFY, and FPF1 gene homologs of the flowering pathway genes of Arabidopsis were analyzed in strawberry. We found that these genes were highly expressed in FvWRKY71-overexpressing transgenic plants. The transcriptional regulatory cascade of FvWRKY71 involved in accelerating flowering in woodland strawberry (Fig. 8) differs from the regulatory mechanism of WRKY71 in promoting flowering in Arabidopsis. FvWRKY71 promotes flowering via the direct modulation of FvFUL, FvSEP1, FvAGL42, FvLFY, and FvFPF1 expression. These data will enrich the known regulatory network of WRKY71 in different plants and provide information on the promotion of flowering on Rosaceae plants.

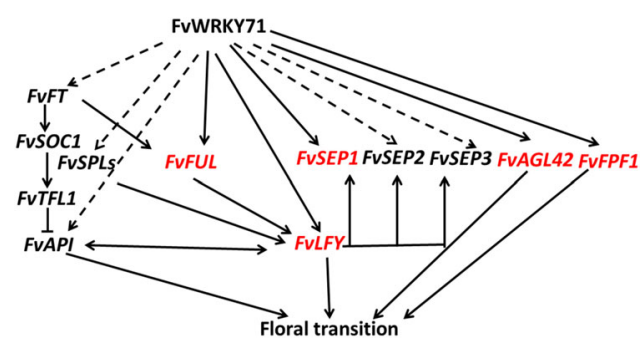

Fig. 8 Proposed transcriptional regulatory cascade whereby FvWRKY71 regulates flowering in woodland strawberry. FVWRKY71 accelerates flowering via the direct activation of FVFUL, FVLFY, FVFPF1, FVAGL42, and FVSEP1 (red font) and the indirect regulation of FvFT, FVSPL3, FVSPL4, FVAP1, FVSEP2, and FVSEP3 (dotted line). The other solid lines indicate regulatory relationships reported in the literature $29,48,51,52,55,59,60$. Arrows represent activation, and bars represent repression 


\section{Materials and methods}

\section{Plant materials and growth conditions}

The material used in this study was the diploid woodland strawberry (Fragaria vesca) 'Ruegen'. The strawberries were grown in the greenhouse at Shenyang Agricultural University, China. FvWRKY71-overexpressing transgenic strawberry plants and wild-type plants were transferred from culture medium to the soil substrate and cultivated in an incubator at $23{ }^{\circ} \mathrm{C}(16 \mathrm{~h} / 8 \mathrm{~h}$, light/dark). Thirty days later, the plants were transplanted to the greenhouse of Shenyang Agricultural University in June. The days to flowering were recorded in the transgenic plants (six for line 3 and six for line 21) and six wild-type plants in the greenhouse after transfer from culture medium.

Wild-type (WT) Arabidopsis (Columbia) seeds were synchronized at $4{ }^{\circ} \mathrm{C}$ for 2 days and then placed at $22^{\circ} \mathrm{C} \pm 2{ }^{\circ} \mathrm{C}$ under long-day ( $16 \mathrm{~h} / 8 \mathrm{~h}$, light/dark) conditions.

The tobacco species used for transient expression analysis was Nicotiana benthamiana, which was grown at $23{ }^{\circ} \mathrm{C}$ under a long-day photoperiod $(16 \mathrm{~h} / 8 \mathrm{~h}$, light/dark) for 1 month.

\section{Phylogenetic analysis and multiple-sequence alignment}

The full-length sequences of WRKY71 proteins from different plants were aligned by using ClustalW (http:// www.clustal.org/), and the phylogenetic tree was constructed using MEGA6.0 software (http://www. megasoftware.net/) ${ }^{61}$. The neighbor-joining (NJ) method was used to produce a phylogenetic tree with the following parameters: bootstrap analysis (1,000 replicates; random seeds), Poisson correction and pairwise deletion $^{61}$. The FvWRKY71 orthologs used for phylogenetic tree construction came from Arabidopsis thaliana, Prunus persica, Malus $x$ domestica, Pyrus bretschneideri, Prunus mume, Prunus avium, Vitis vinifera, Theobroma cacao, Gossypium arboreum, and Carica papaya. DNAMAN software was used to carry out amino acid sequence alignments between FvWRKY71 and different species (version 6.0; Lynnon Biosoft, Quebec, Canada).

\section{Subcellular localization}

The coding region without the stop codon of FvWRKY71 was amplified by RT-PCR from the red fruit of 'Ruegen' based on woodland strawberry genome information ${ }^{62,63}$. The FvWRKY71-F and FvWRKY71-R primers are shown in Supplemental Table S1. The products were inserted into the pRI101-GFP vector with EcoRI and KpnI to generate the plasmid p35S::FvWRKY71-GFP. The pRI101-GFP and p35S:: FvWRKY71-GFP plasmids were transformed into Agrobacterium tumefaciens strain EHA105. The leaves of 1month-old tobacco $(N$. benthamiana) plants were infiltrated with $A$. tumefaciens cells harboring pRI101-GFP and pRI101FvWRKY71-GFP. The GFP fluorescence signal was observed by confocal fluorescence microscopy (Leica DMi8 A, Wetzlar, Germany) 2 days after transient transformation.

\section{Transactivation assay}

The coding region of FvWRKY71 was amplified by RTPCR from the red fruit of 'Ruegen' using specific primers (Supplemental Table S1) and then inserted into the pGBT9 vector. The pGBT9-FvWRKY71 plasmid and the pGBT9 vector were introduced into $\mathrm{Y} 2 \mathrm{H}$ Gold cells using the PEG/ LiAc method ${ }^{64}$, and the cells were grown on SD/-Trp agar plates. The pGBT9 vector was used as a negative control. After 3 days, single transformed yeast colonies were diluted with $10 \mu \mathrm{L}$ sterile water, and a $3-\mu \mathrm{L}$ aliquots were dropped onto SD/-Trp and SD/-Trp/-Leu/-Ade/X-a-Gal plates to observe yeast growth at $30^{\circ} \mathrm{C}$ for $3-4$ days. Next, we amplified the coding sequences of the $\mathrm{N}$-terminus and $\mathrm{C}$ terminus of FvWRKY71 by RT-PCR from the red fruit of 'Ruegen' (Supplemental Table S1), and the PCR products were cloned into the pGBT9 vector to identify the activation area at the $\mathrm{N}$-terminus or $\mathrm{C}$-terminus.

\section{Overexpression vector construction and plant transformation}

The full-length coding region of FvWRKY71 was amplified by RT-PCR from the red fruit of 'Ruegen' and cloned into pRI101-AN to generate the overexpression plasmid p35S::FvWRKY71. The PCR primer sets are shown in Supplemental Table S1. The placsmid was confirmed by sequencing. The plasmid was introduced into $A$. tumefaciens strain GV3101 via the freeze-thaw method and transformed into Arabidopsis via the floral dip method ${ }^{65}$. Positive transgenic plants were screened on plates containing 1/2 MS medium with $30 \mathrm{mg} / \mathrm{L}$ kanamycin. Furthermore, two $\mathrm{T}_{3}$ generation transgenic Arabidopsis lines (Line 1 and Line 5) were detected by RT-qPCR for gene expression analysis. The numbers of rosette leaves were analyzed in each plant after the first inflorescence emergence. Ten plants of each line were used for analyzing the numbers of rosette leaves.

To further elucidate the function of FvWRKY71, we introduced the overexpression plasmid p35S::FvWRKY71 into 'Ruegen' strawberry as described previously ${ }^{66}$. Explants were placed on medium supplemented with $250 \mathrm{mg} / \mathrm{L}$ timetin and $250 \mathrm{mg} / \mathrm{L}$ cefotaxime and $10 \mathrm{mg} / \mathrm{L}$ kanamycin for selection culture in the dark. When adventitious buds appeared, they were placed under light. The concentration of kanamycin was increased from 10 to $30 \mathrm{mg} / \mathrm{L}$ in subsequent subcultures. After $\sim 8$ months of selective culture, we obtained transgenic plants and transferred them to the greenhouse, where they were grown under short-day conditions. The positive transgenic strawberry plants were evaluated by PCR to detect DNA levels and RT-qPCR for gene expression analysis. 


\section{RNA isolation and expression analysis}

To test the expression of representative genes involved in flowering, young leaves of the transgenic plants and wild-type plants were chosen. Total RNA was isolated from three mixed samples using the modified CTAB method as described previously ${ }^{67}$. Each mixed sample was obtained from six transgenic plants or wild-type plants. Total RNA $(1 \mu \mathrm{g})$ was reverse-transcribed in a $20-\mu \mathrm{L}$ reaction using a PrimeScript ${ }^{\mathrm{TM}} \mathrm{RT}$ reagent Kit (Takara, Japan). The $10-\mu \mathrm{L}$ reaction for removing genomic DNA contained $2.0 \mu \mathrm{L}$ of $5 \times$ gDNA Eraser Buffer, $1.0 \mu \mathrm{L}$ gDNA Eraser, $1 \mu \mathrm{g}$ RNA, and RNase-free $\mathrm{ddH}_{2} \mathrm{O}$ to $10 \mu \mathrm{L}$. The $20-\mu \mathrm{L}$ reverse transcription reaction contained $10 \mu \mathrm{L}$ of the previous reaction product, $1.0 \mu \mathrm{L}$ PrimeScript RT Enzyme Mix1, $1.0 \mu \mathrm{L}$ RT Primer Mix, $4.0 \mu \mathrm{L}$ of $5 \times$ PrimeScript Buffer 2, and $4.0 \mu \mathrm{L}$ of RNase-free $\mathrm{ddH}_{2} \mathrm{O}$. The following thermal profile was used for reverse transcription: $42^{\circ} \mathrm{C}$ for $5 \mathrm{~min}$, followed by $37^{\circ} \mathrm{C}$ for $30 \mathrm{~min}$ and $85^{\circ} \mathrm{C}$ for $5 \mathrm{~s}$. RT-qPCR was performed in an ABI 7500 system (Applied Biosystems, Foster City, CA, USA). All cDNA samples were diluted with $\mathrm{ddH}_{2} \mathrm{O}(\mathrm{V}: \mathrm{V} ; 1: 4)$. RT-qPCR was performed in a volume of $10 \mu \mathrm{L}$ containing $5 \mu \mathrm{L}$ UltraSYBR Green Mixture reagent (ComWin Biotech, Beijing), $0.5 \mu \mathrm{L} \mathrm{cDNA}, 1 \mu \mathrm{L}$ of the primer set, and

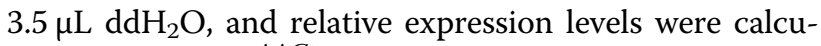
lated using the $2^{-\Delta \Delta \mathrm{Ct}}$ method, taking the WT plants as a control. Each sample was quantified in triplicate with three biological replicates. The reaction conditions for RT-qPCR consisted of an initial step $\left(50^{\circ} \mathrm{C}\right.$ for $2 \mathrm{~min}$, $95^{\circ} \mathrm{C}$ for $\left.10 \mathrm{~min}\right)$, followed by 40 cycles $\left(95^{\circ} \mathrm{C}\right.$ for $15 \mathrm{~s}$, $60^{\circ} \mathrm{C}$ for $\left.1 \mathrm{~min}\right)$ and a denaturing step $\left(95^{\circ} \mathrm{C}\right.$ for $15 \mathrm{~s}$, $60^{\circ} \mathrm{C}$ for $15 \mathrm{~s}, 95^{\circ} \mathrm{C}$ for $15 \mathrm{~s}$ ). We performed normalization first with Arabidopsis $18 \mathrm{~S}$ rRNA as a control and then with strawberry $26 \mathrm{~S}$ rRNA as a control ${ }^{68}$. The gene primers used for the RT-qPCR experiments are listed in Supplemental Table S1.

\section{Yeast one-hybrid assay}

The coding sequence of FvWRKY71 was obtained through RT-PCR with specific primers from the red fruit of 'Ruegen'. Then, the fragment was digested with EcoRI and $B a m H I$ and inserted into the pGAD424 vector to obtain pAD-FvWRKY71. The promoter regions of FvFUL, $F v S E P 1, F v A G L 42, F v L F Y, F v F P F 1$, and FvSPL4 were amplified by PCR from the genomic DNA of the leaves of 'Ruegen', verified by sequencing and cloned into the pAbAi vector with the EcoRI and BamHI restriction enzymes. The primers used for the yeast one-hybrid analysis are listed in Supplemental Table S1.

The plasmids proFUL-pAbAi, proSEP1-pAbAi, proA GL42-pAbAi, proLFY-pAbAi, proFPF1-pAbAi, and proSPL4-pAbAi were introduced into the $\mathrm{Y} 1 \mathrm{H}$ Gold yeast strain using the PEG/LiAC method ${ }^{61}$ and grown on SD/Ura agar plates. After 3 days, we transferred positive yeast to agar plates containing SD/-Ura/+AbA $(50-500 \mu \mathrm{g} / \mathrm{L})$ and conducted screening to determine the minimum inhibitory concentration of $\mathrm{AbA}$ in relation to yeast growth. Next, we transformed pAD-FvWRKY71 into yeast strains containing proFUL-pAbAi, proSEP1-pAbAi, proAGL42-pAbAi, proLFY-pAbAi, proFPF1-pAbAi, and proSPL4-pAbAi and plated them in SD/-Leu/and SD/Leu/+AbA plates. Finally, we observed the growth of the yeast on agar plates. The Yeast Protocols Handbook (Clontech) was used to perform the above yeast experiments.

\section{Dual-luciferase reporter system}

Two kilobase sequences of the FvFUL, FvSEP1, $F v A G L 42, F v L F Y, F v F P F 1$, and FvSPL4 promoters containing W-boxes (TTGACT/C) were amplified from the genomic DNA of the leaves of 'Ruegen'. The fragments were digested with HindIII and $\mathrm{BamHI}$ and inserted into the pGreenII0800-LUC vector as reporter plasmids ${ }^{35}$. The overexpression vector p35S::FvWRKY71 was used as an effector plasmid.

These plasmids were introduced into $A$. tumefaciens strain GV3101 via the freeze-thaw method. Agrobacterium strain GV3101 carrying the effector plasmid (empty vector or p35S::FvWRKY71) and the specific reporter plasmid (ProFvFUL::LUC, ProFvSEP1::LUC, ProFvAGL 42::LUC, ProFvLFY::LUC, ProFvFPF1::LUC, and ProF vSPL4::LUC) was cultured to $\mathrm{OD}_{600}=1.0$ at $28^{\circ} \mathrm{C}$. The cells were harvested and resuspended in medium $(1 \mathrm{M}$ $\mathrm{MgCl}_{2}, 100 \mathrm{mM}$ acetosyringone and $1 \mathrm{M} \mathrm{MES}, \mathrm{pH}$ 5.6). Then, the solution was placed at room temperature without shaking for $2 \mathrm{~h}$ and infiltrated into 1-month-old $N$. benthamiana leaves. The infiltrated tobacco plant was placed in the dark for $24 \mathrm{~h}$ and then placed under light for $48 \mathrm{~h}$. We tested luciferase signaling with a living fluorescence imager (Lb985, Berthold, Germany). The transcriptional activity in the infiltrated tobacco leaves indicated by the ratio of LUC to REN was investigated with a dual luciferase reporter gene assay kit (Beyotime, China). Six biological repeats were measured for each sample. The primers used for the LUC/REN activity analysis are listed in Supplemental Table S1.

\section{Accession numbers}

AT5G62165 (AGL42), AT1G69120 (AP1), AT5G24860 (FPF1), AT1G65480 (FT), AT5G60910 (FUL), AT5G61850 (LFY), AT5G15800.2 (SEP1), AT3G02310 (SEP2), AT1G2 4260 (SEP3), AT2G33810 (SPL3), AT1G53160 (SPL4), FvH 4_7g28740.1 (AGL42), FvH4_4g29600.1 (AP1), FvH4_2g3 0770.1 (FPF1), FvH4_6g00090.1 (FT), FvH4_5g13500.1 (FUL), FvH4_5g09660.1 (LFY), FvH4_7g28690.1 (SEP1), FvH 4_6g46420.1 (SEP2), FvH4_4g23530.1 (SEP3), FvH4_3g08 880 (SPL3), FvH4_3g19650.1 (SPL4), and FvH4_7g12700.1 (SOC1). GenBank: JN172097.1 (FvTFL1), 


\section{Acknowledgements}

We thank Yuepeng Han from the Wuhan Botanical Garden of the Chinese Academy of Sciences for kindly providing the pGreenll10800-LUC vector. This work was supported by the National Key R\&D Program of China (2019YFD1000200), National Natural Science Foundation of China (31601730), China Postdoctoral Science Foundation (2017M611264), Key R\&D and Technology Transfer Program (Z17-0-035), Shenyang Young and Middle-aged Science and Technology Innovation Talents Support Plan (RC190446) and LiaoNing Revitalization Talents Program (XLYC1902069).

\section{Author contributions}

Y.L. and J.Z. designed the experiments. Y.S., Y.L., B.W., H.D., and S.Y conducted the experiments. J.Z., H.L., and Y.L. analyzed the data. Y.S., Y.L., Z.Z., and J.Z. wrote and modified the manuscript. All authors involved in this study read and approved the manuscript.

\section{Conflict of interest}

The authors declare that they have no conflict of interest.

Supplementary Information accompanies this paper at (https://doi.org/ 10.1038/s41438-020-00355-4)

Received: 9 January 2020 Revised: 14 May 2020 Accepted: 16 June 2020 Published online: 01 September 2020

\section{References}

1. Ishiguro, R. \& Nakamura, K. Characterization of a cDNA encoding a novel DNAbinding protein, SPF1, that recognizes SP8 sequences in the $5^{\prime}$ upstream regions of genes coding for sporamin and $\beta$-amylase from sweet potato. Mol. Gen. Genet. 244, 563-571 (1994).

2. Rushton, P. J., Somssich, I. E., Patricia, R. \& Shen, Q. J. WRKY transcription factors. Plant Signal. Behav. 15, 247-258 (2010).

3. Bakshi, M. \& Oelmüller, R. WRKY transcription factors: Jack of many trades in plants. Plant Signal. Behav. 9, e27700 (2014).

4. Banerjee, A. \& Roychoudhury, A. WRKY proteins: signaling and regulation of expression during abiotic stress responses Scientific World J. 2015, 807560 (2015).

5. Zheng, Z. et al. Arabidopsis WRKY33 transcription factor is required for resistance to necrotrophic fungal pathogens. Plant J. 48, 592-605 (2010).

6. Birkenbihl, R. P., Diezel, C. \& Somssich, I. E. Arabidopsis WRKY33 is a key transcriptional regulator of hormonal and metabolic responses toward Botrytis cinerea infection. Plant Physiol. 159, 266-285 (2012).

7. Hu, Y., Dong, Q. \& Yu, D. Arabidopsis WRKY46 coordinates with WRKY70 and WRKY53 in basal resistance against pathogen Pseudomonas syringae. Plant Sci. 185-186, 288-297 (2012).

8. Jiang, Y. \& Deyholos, M. Functional characterization of Arabidopsis $\mathrm{NaCl}$ inducible WRKY25 and WRKY33 transcription factors in abiotic stresses. Plant Mol. Biol. 69, 91-105 (2009).

9. Ren, $X$. et al. $A B O 3$, a WRKY transcription factor, mediates plant responses to abscisic acid and drought tolerance in Arabidopsis. Plant J. 63, 417-429 (2010).

10. Li, S. et al. Arabidopsis thaliana WRKY25, WRKY26, and WRKY33 coordinate induction of plant thermotolerance. Planta 233, 1237-1252 (2011).

11. $\mathrm{Hu}, \mathrm{Y}$. et al. Arabidopsis transcription factor WRKY8 functions antagonistically with its interacting partner VQ9 to modulate salinity stress tolerance. Plant J. 74, 730-745 (2013).

12. Silke, R. \& Imre, E. S. Targets of AtWRKY6 regulation during plant senescence and pathogen defense. Genes Dev. 16, 1139-1149 (2002).

13. Wang, $\mathrm{H}$. et al. Mutation of WRKY transcription factors initiates pith secondary wall formation and increases stem biomass in dicotyledonous plants. Proc. Natl Acad. Sci. USA 107, 22338-22343 (2010).

14. Guo, D. et al. The WRKY transcription factor WRKY71/EXB1 controls shoot branching by transcriptionally regulating RAX genes in Arabidopsis. Plant Cell. 27, 3112-3127 (2015)

15. Liu, J. et al. Alternative splicing of rice WRKY62 and WRKY76 transcription factor genes in pathogen defense. Plant Physiol. 171 1427-14429 (2016).
16. Qiu, D. et al. OsWRKY13 mediates rice disease resistance by regulating defense-related genes in salicylate- and jasmonate-dependent signaling. Mol. Plant Microbe Interact. 20, 492-499 (2007).

17. Qiu, Y. \& Yu, D. Over-expression of the stress-induced OsWRKY45 enhances disease resistance and drought tolerance in Arabidopsis. Environ. Exp. Bot. 65, 35-47 (2009).

18. Wu, X. et al. Enhanced heat and drought tolerance in transgenic rice seedlings overexpressing OsWRKY11 under the control of HSP101 promoter. Plant Cell Rep. 28, 21-30 (2009)

19. Zhang, L. et al. Transcription factor WRKY75 interacts with DELLA proteins to affect flowering. Plant Physiol. 176, 790-803 (2018).

20. Li, W. et al. Arabidopsis WRKY transcription factors WRKY12 and WRKY13 oppositely regulate flowering under short-day conditions. Mol. Plant 9 1492-1503 (2016)

21. Yu, Y. et al. MIWRKY12, a novel Miscanthus transcription factor, participates in pith secondary cell wall formation and promotes flowering. Plant Sci. 212, 1-9 (2013).

22. Yu, Y. et al. WRKY71 accelerates flowering via the direct activation of FLOWERING LOCUS T and LEAFY in Arabidopsis thaliana. Plant J. 85 96-106 (2016)

23. Huang, R. W. et al. CPWRKY71, a WRKY transcription factor gene of Wintersweet (Chimonanthus praecox), promotes flowering and leaf senescence in Arabidopsis. Int. J. Mol. Sci. 20, 5325 (2019).

24. Darrow, G. The Strawberry: History, Breeding and Physiology. (Holt, Rinehart and Winston, NY, 1966).

25. Rousseau-Gueutin, M. et al. Comparative genetic mapping between octoploid and diploid Fragaria species reveals a high level of colinearity between their genomes and the essentially disomic behavior of the cultivated octoploid strawberry. Genetics 179, 2045-2060 (2008).

26. Nakajima, R. et al. Molecular cloning and expression analyses of FaFT, FaTFL, and FaAP1 genes in cultivated strawberry: their correlation to flower bud formation. Biol. Plant. 58, 641-648 (2014).

27. Whitaker, V. M. et al. A roadmap for research in octoploid strawberry. Hortic Res. 7, 33 (2020)

28. Brown, T. et al. Genetical control of everbearing habit and 3 other characters in varieties of Fragaria vesca. Euphytica 14, 97-112 (1965).

29. Koskela, E. A. et al. Mutation in TERMINAL FLOWER1 reverses the photoperiodic requirement for flowering in the wild strawberry Fragaria vesca. Plant Physiol. 159, 1043-1054 (2012).

30. Mouhu, $K$. et al. The Fragaria vesca homolog of SUPPRESSOR OF OVEREXPRESSION OF CONSTANS1 represses flowering and promotes vegetative growth. Plant Cell. 25, 3296-3310 (2013).

31. Gaston, A. et al. PFRU, a single dominant locus regulates the balance between sexual and asexual plant reproduction in cultivated strawberry. J. Exp. Bot. 64 1837-1848 (2013).

32. Zhou, H. et al. Genome-wide analysis of the expression of wrky family genes in different developmental stages of wild strawberry (Fragaria vesca) fruit. PLOS ONE 11, e0154312 (2016).

33. Wei, W. et al. The WRKY transcription factors in the diploid woodland strawberry Fragaria vesca: Identification and expression analysis under biotic and abiotic stresses. Plant Physiol. Biochem. 105, 129-144 (2016).

34. Eulgem, T., Rushton, P. J., Robatzek, S. \& Somssich, I. E. The WRKY superfamily of plant transcription factors. Trends Plant Sci. 5, 199-206 (2000).

35. Hellens, R. P. et al. Transient expression vectors for functional genomics, quantification of promoter activity and RNA silencing in plants. Plant Methods 1, 13 (2005).

36. Richard, A. Seasonal and developmental timing of flowering. Plant J. 61 , 1001-1013 (2010)

37. $\mathrm{Gu}, \mathrm{X}$. et al. Arabidopsis FLC clade members form flowering-repressor complexes coordinating responses to endogenous and environmental cues. Nat Commun. 4, 1947 (2013).

38. Amasino, R. M. \& Michaels, S. D. The timing of flowering. Plant Physiol. 154 516-520 (2010).

39. Andrés, F. \& Coupland, G. The genetic basis of flowering responses to seasonal cues. Nat. Rev. Genet. 13, 627-639 (2012).

40. Wang, J. W. Regulation of flowering time by the miR156-mediated age pathway. J. Exp. Bot. 65, 4723-4730 (2014).

41. Mouhu, K. et al. Identification of flowering genes in strawberry, a perennial SD plant. BMC Plant. Biol. 9, 122 (2009). 
42. Hu, J. Y. \& Meaux, J. D. miR824-regulated AGAMOUS-LIKE16 contributes to flowering time repression in Arabidopsis. Plant Cell $\mathbf{2 6}$ 2024-2037 (2014)

43. Hartmann, U. et al. Molecular cloning of SVP: a negative regulator of the floral transition in Arabidopsis. Plant J. 21, 351-360 (2010).

44. Markus, S. et al. Dissection of floral induction pathways using global expression analysis. Development 130, 6001-6012 (2003).

45. Cao, S. et al. A distal CCAAT/NUCLEAR FACTOR Y complex promotes chromatin looping at the FLOWERING LOCUS T promoter and regulates the timing of flowering in Arabidopsis. Plant Cell 26, 1009-1017 (2014).

46. Wang, C. Q. et al. BBX19 interacts with CONSTANS to repress FLOWERING LOCUS T transcription, defining a flowering time checkpoint in Arabidopsis. Plant Cell 26, 3589-3602 (2014).

47. Sawa, M. \& Kay, S. A. GIGANTEA directly activates FLOWERING LOCUS $T$ in Arabidopsis thaliana. Proc. Natl Acad. Sci. USA 108, 11698-11703 (2011).

48. Wickland, D. P. \& Hanzawa, Y. The FLOWERING LOCUS T/TERMINAL FLOWER 1 gene family: functional evolution and molecular mechanisms. Mol. Plant 8 , 983-997 (2015).

49. Blázquez, M. A. et al. LEAFY expression and flower initiation in Arabidopsis. Development 124, 3835-3844 (1997).

50. Ferrándiz, $C$. et al. Redundant regulation of meristem identity and plant architecture by FRUITFULL, APETALA1 and CAULIFLOWER. Development 127, 725-734 (2000).

51. Mandel, M. A. \& Yanofsky, M. F. A gene triggering flower formation in Arabidopsis. Nature 377, 522-524 (1995).

52. Iwata, $\mathrm{H}$. et al. The TFL1 homologue KSN is a regulator of continuous flowering in rose and strawberry. Plant J. 69, 116-125 (2012).

53. Randoux, M. et al. Gibberellins regulate the transcription of the continuous flowering regulator, RoKSN, a rose TFL 1 homologue. J. Exp. Bot. 63, 6543-6554 (2012).

54. Rantanen, M. et al. Strawberry homolog of TERMINAL FLOWER1 integrates photoperiod and temperature signals to inhibit flowering. Plant J. 82, 163-173 (2015).

55. Melzer, S. et al. FPF1 modulates the competence to flowering in Arabidopsis. Plant J. 18, 395-405 (1999).
56. Yamaguchi, A. \& Wu, M. L. The microRNA-regulated SBP-Box transcription factor SPL3 is a direct upstream activator of LEAFY, FRUITFULL and APETALA1. Dev. Cell 17, 268-278 (2009)

57. Becker, A. \& Theiben, G. The major clades of MADS-box genes and their role in the development and evolution of flowering plants. Mol. Phylogenet. Evol. 29, 464-489 (2003)

58. Dornelas, M. C. et al. MADS: the missing link between identity and growth? Trends Plant Sci. 16, 89-97 (2011)

59. Pelaz, S. et al. B and C floral organ identity functions require SEPALLATA MADSbox genes. Nature 405, 200-203 (2000).

60. Dorca-Fornell, C., Gregis, V. \& Grand., V. The Arabidopsis SOC1-like genes AGL42, AGL71 and AGL72 promote flowering in the shoot apical and axillary meristems. Plant J. 67, 1006-1017 (2011).

61. Tamura, $\mathrm{K}$. et al. MEGA6: molecular evolutionary genetics analysis version 6.0 Mol. Biol. Evol. 30, 2725-2729 (2013).

62. Shulaev, V. et al. The genome of woodland strawberry (Fragaria vesca). Nat Genet. 43, 109-116 (2011).

63. Edger, P. P. et al. Single-molecule sequencing and optical mapping yields an improved genome of woodland strawberry (Fragaria vesca) with chromosome-scale contiguity. Gigascience 7, gix124 (2017).

64. Gietz, R. D. \& Schiest, R. H. High-efficiency yeast transformation using the LiAC/ SS carrier DNA/PEG method. Nat. Protoc. 2, 31-34 (2007).

65. Clough, S. J. \& Bent, A. F. Floral dip: a simplified method for Agrobacterium-mediated transformation of Arabidopsis thaliana. Plant J. 16, 735-743 (2010).

66. Li, W. J. et al. FveRGA1, encoding a DELLA protein, negatively regulates runner production in Fragaria vesca. Planta 247, 941-951 (2018).

67. Chang, L. et al. Detection of strawberry RNA and DNA viruses by RTPCR using total nucleic acid as a template. J. Phytopathol. 155 431-436 (2010).

68. Livak, K. J. \& Schmittgen, T. D. Analysis of relative gene expression data using real-time quantitative PCR and the $2^{-\Delta \Delta C T}$ Method. Methods 25, 402-408 (2001). 